\title{
Analysis on the Relationship Between Ultraviolet Radiation Characteristics and Asphalt Pavement Disease in Tibet Plateau
}

\author{
Zhang Ning \\ College of Civil Engineering, Qinzhou University, Qinzhou, China
}

\section{Email address:}

linzhijiaotong@126.com

\section{To cite this article:}

Zhang Ning. Analysis on the Relationship Between Ultraviolet Radiation Characteristics and Asphalt Pavement Disease in Tibet Plateau. American Journal of Civil Engineering. Vol. 4, No. 6, 2016, pp. 367-371. doi: 10.11648/j.ajce.20160406.24

Received: November 15, 2016; Accepted: December 2, 2016; Published: December 9, 2016

\begin{abstract}
Through the analysis of the monitoring data of ultraviolet radiation in Tibet, and compared with the related areas, the distribution and intensity characteristics of ultraviolet radiation in Tibet plateau were obtained. Tibet Plateau radiation intensity equivalent to about three times more than the same latitude as the southern region, and also much higher than in eastern Tibet, the solar ultraviolet radiation is the strongest region in the world. The survey summarized the main characteristics of Tibetan asphalt pavement disease, analysed the strong ultraviolet irradiation, the relationship between Tibetan road disease and strong ultraviolet radiation, and obtained the general rule of the damage of asphalt pavement in Tibetan: ultraviolet radiation $\rightarrow$ asphalt pavement whitened $\rightarrow$ asphalt hardening $\rightarrow$ micro cracks appeared $\rightarrow$ crack expansion $\rightarrow$ cracking $\rightarrow$ water $\rightarrow$ asphalt peeling (loose), pit slot etc. The origin of this Law in the Tibetan strong ultraviolet radiation, High ultraviolet radiation causes aging of asphalt, losing its original performance, which led to destruction of roads in climate and traffic load, therefore, to strengthen the anti-aging properties of asphalt will be greatly improved Tibet District road usage.
\end{abstract}

Keywords: Plateau, Ultraviolet, Asphalt Pavement, Disease

\section{Introduction}

Tibetan Plateau due to low latitude, high altitude, clean air, is the largest solar radiation area in China, up to $6700 \sim$ $9200 \mathrm{MJ} / \mathrm{m}^{2}$, the total amount of radiation increases with increasing height above sea level, altitude impact greater than the impact of latitude; The average annual total radiation of Tibetan Plateau is $2512 \sim 3350 \mathrm{MJ} / \mathrm{m}^{2}$, the largest in the whole country, and the average annual rate is positive [1]. At an altitude of $5000 \mathrm{~m}$ the following areas, the total amount of the radiation balance of same latitude from east to west with increasing altitude increases, the $98.8 \%$ of total radiation balance of the amount of heat consumed in evaporation and heat of atmospheric turbulence.

The solar radiation is the source of heat absorption of the highway asphalt pavement on the Tibet Plateau. The sun's ultraviolet radiation is also very high, studies have shown that the Tibet plateau accept the UV radiation is three times the same latitude plains above, and it is the main factor for early aging of asphalt pavement in Tibet plateau [2], [3], [4].
Therefore, the study of Tibetan plateau ultraviolet radiation characteristics and analysis of asphalt pavement failure characteristics has important implications for asphalt pavement construction in Tibet and anti-aging design. This paper by UV existing monitoring data and comparative analysis of Tibet and other regions to study the regional distribution and intensity of the ultraviolet features, combined with Tibetan road damage phenomena to discuss their mutual relations and provide the basis for the Tibetan plateau asphalt pavement anti ultraviolet aging design.

\section{Tibet Plateau Ultraviolet Observation and Analysis Features}

In recent years, with the research of the Tibet Plateau ultraviolet, Tibet has established a plurality of ultraviolet observation points, made a large number of observational data, In this paper, the data is taken from the Lhasa Meteorological Bureau observation point (elevation 3658m). Also select similar latitude in Chongqing, Wuhan, Shanghai, 
Rong monastery, Shiquanhe and large difference in the Beijing area monitoring data contrast to analyze the Tibetan plateau ultraviolet distribution and strength characteristics.

\subsection{Analysis of Solar Ultraviolet Radiation Law in Lhasa City}

\subsubsection{The Monthly Variation of Ultraviolet Radiation}

Based on monitoring data in March 2013, April, May 22nd ultraviolet radiation per hour were analyzed for three consecutive months of 22 , are in fine weather. The results of observation can accurately reflect the Lhasa city each month of radiation. Monitoring data variation as shown in Figure 1.

As can be seen from Figure 1: From March to May, the gradual increase of the amount of ultraviolet radiation, every day from eight o'clock to begin about two p.m. peaked after attenuation in the evening seven p.m. end, the whole process sinusoidal changes; having a significant cyclical, only in the next cycle of UV radiation than the previous cycle has been strengthened, according to the research: Tibet in August, the strongest ultraviolet radiation, then gradually decay, according to the results of continuous observations: in 2013, the total amount of ultraviolet radiation in Lhasa, 361.9MJ / $\mathrm{m}^{2}$, is 2.5 times more than the same latitude as the Chongqing area.

Table 1 The situation of solar radiation in Lhasa and the main stations in China is presented. The annual total solar radiation in Lhasa is more than that in the mainland, which is about twice as many as that in the eastern part of the same latitude. Tibetan monastery Rong up to $8769 \mathrm{MJ} / \mathrm{m}^{2}$, followed by Tibet Shiquanhe. According to the observation data of Lhasa station in thirty-three years, the annual variation of total radiation in Lhasa is small, and the average relative change rate is only $4.2 \%$, compared with the same latitude in the eastern region to be small, but slightly larger than the Sichuan Basin. Seasonal variation around all places solar radiation has obvious climate characteristics, the maximum summer, spring, followed by winter minimum, In Lhasa, for example, spring, summer, autumn and winter seasons and the amount of radiation than in the total amount of radiation is respectively $27.7 \%, 29.5 \%, 23.4 \%$ and $19.3 \%$.

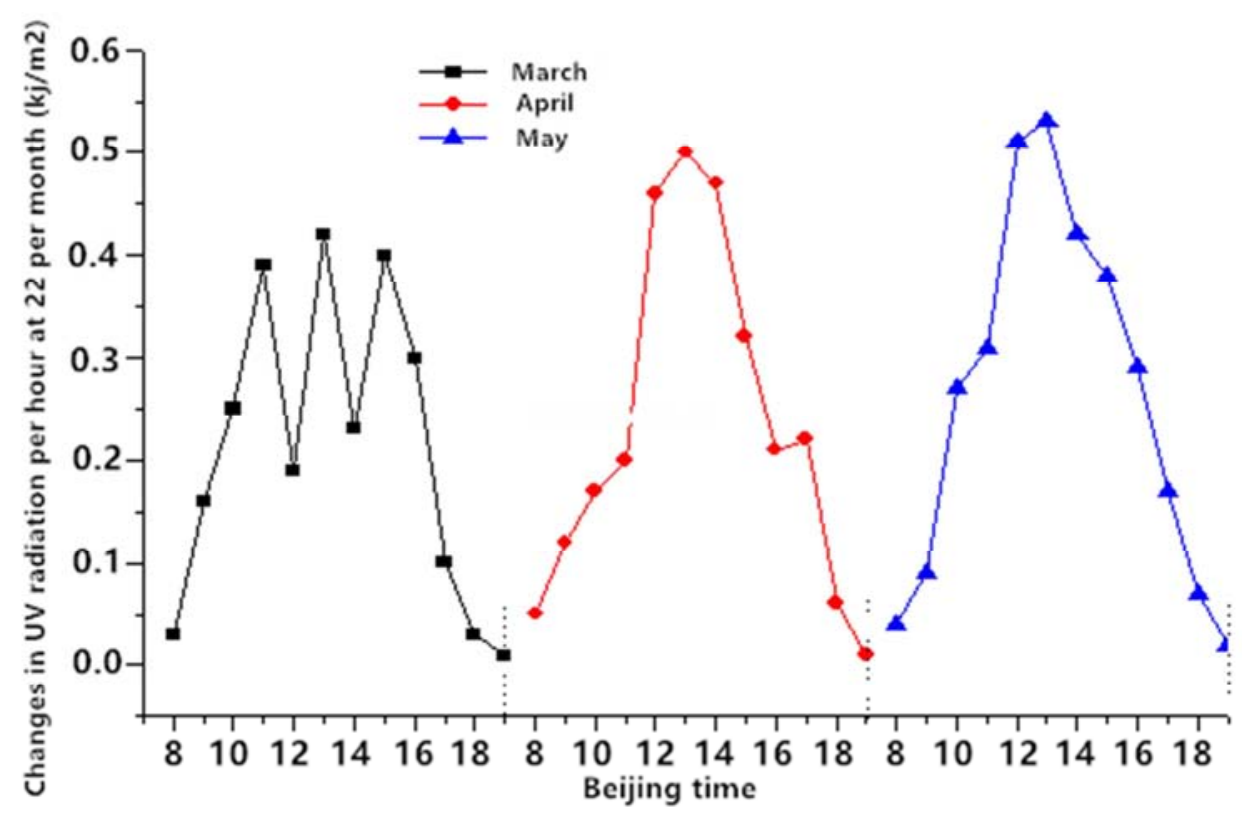

Fig. 1. Changes in March 2013, April, May Lhasa ultraviolet radiation per hour.

Table 1. Comparison of the solar total radiation throughout the year.

\begin{tabular}{lll}
\hline Site name & latitude & The total amount of radiation $\left(\mathbf{M J} / \mathbf{m}^{\mathbf{2}}\right)$ \\
\hline Lhasa & $29^{\circ} 42^{\prime}$ & 7924 \\
Shiquanhe & $32^{\circ} 30^{\prime}$ & 8102 \\
Rong monastery & $31^{\circ} 25^{\prime}$ & 8769 \\
Chongqing & $29^{\circ} 31^{\prime}$ & 3499 \\
Wuhan & $30^{\circ} 38^{\prime}$ & 4711 \\
Beijing & $39^{\circ} 48^{\prime}$ & 5200 \\
Shanghai & $31^{\circ} 07^{\prime}$ & 4573 \\
\hline
\end{tabular}

\subsubsection{Distribution Characteristics of Radiation Energy of} Solar Radiation

According to the observation of Lhasa station (altitude $3688 \mathrm{~m}$ ), daily total solar radiation into light observation data calculated six bands of light radiation relative flux $\eta_{\Delta \lambda}(\Delta \lambda$ ratio of total solar radiation and total solar radiation, take $\%$ as unit) of the monthly average values, see Table 2 as shown in table from 1999 April 15 to October 15. From table 2, it is seen that daily average relative fluxes of the optical radiation $\eta_{\Delta \lambda}$ from April to October: ultraviolet (292 400nm) 5.3\%, visible (400 693nm) 42.8\%, infrared (693 3000nm) 51.9\%. There were also differences in the relative flux of the light radiation $\eta_{\Delta \lambda}$ between the moon and the moon. The relative flux of the ultraviolet band was the largest in June and decreased rapidly in the future. Table 3 relative fluxes of light radiation between Lhasa and Beijing in June and September. Seen from the table 2 , Lhasa's ultraviolet band $\eta_{\triangle \lambda}$ are higher than Beijing two percentage points, while the calculation results show that the total amount of ultraviolet radiation in Beijing is more than two times the Lhasa area. 
Table 2. Relative flux of solar radiation $\eta_{\Delta \lambda}(\%)$ monthly mean value.

\begin{tabular}{|c|c|c|c|c|c|c|c|c|}
\hline \multirow{2}{*}{$\operatorname{Color}\left(\Delta_{\lambda}(\mathbf{n m})\right)$} & \multicolumn{8}{|c|}{ Month } \\
\hline & 4 & 5 & 6 & 7 & 8 & 9 & 10 & Average \\
\hline Ultraviolet (292 400) & 5.6 & 6.0 & 6.4 & 5.6 & 5.5 & 5.4 & 4.7 & 5.6 \\
\hline Blue violet (400 488) & 14.8 & 14.5 & 14.7 & 15.0 & 15.7 & 14.2 & 13.3 & 14.6 \\
\hline Green light (488 522) & 5.5 & 5.7 & 5.5 & 5.5 & 5.1 & 6.2 & 6.5 & 5.7 \\
\hline Yellow orange (522 627) & 16.9 & 16.9 & 16.9 & 16.8 & 17.5 & 17.7 & 16.3 & 17.0 \\
\hline Red light (627 693) & 4.9 & 5.2 & 5.3 & 5.1 & 5.3 & 5.4 & 5.1 & 5.2 \\
\hline Infrared $(693 \sim 3000)$ & 52.2 & 51.2 & 51.8 & 51.9 & 50.9 & 51.0 & 54.1 & 51.9 \\
\hline
\end{tabular}

Table 3. Comparison of monthly average value of solar radiation $\eta_{\Delta \lambda}(\%)$ between Lhasa and Beijing.

\begin{tabular}{lllllllll}
\hline \multirow{2}{*}{ Time } & \multirow{2}{*}{ Location } & Spectrum & & & & \\
\cline { 3 - 8 } & & ultraviolet & Blue violet & Green & Yellow orange & Red & infra-red & visible light \\
\hline \multirow{2}{*}{ June } & Lhasa & 6.4 & 14.7 & 5.5 & 16.9 & 5.3 & 51.2 & 42.4 \\
& Beijing & 4.4 & 11.6 & 12.6 & 14.9 & 9.0 & 47.4 & 48.1 \\
\multirow{2}{*}{ September } & Lhasa & 5.4 & 14.2 & 6.2 & 16.0 & 5.1 & 53.1 & 44.2 \\
& Beijing & 3.9 & 9.9 & 12.6 & 15.4 & 9.1 & 48.1 & 47.0 \\
\hline
\end{tabular}

\subsection{Comparative Analysis of the Solar Ultraviolet Radiation and the Surrounding Cities of Lhasa City}

Figure 2 shows the average value of UV-B radiation in the cities around the city of Lhasa and the Tibetan Plateau in May to August. The blue line is Shiquanhe average value. Furthermore, in various cities, Shiquanhe UV-B radiation is the highest with the little change; the UV-B radiation in Chengdu is the lowest and less than half of the Shiquanhe. The amount of UV-B radiation in Lhasa is just lower than the Shiquanhe, which ranked second. However, UV-B radiation in Lhasa varied greatly in different years. Although Nagqu altitude $(4468 \mathrm{~m})$ is higher than the altitude of Lhasa $3678 \mathrm{~m}$, ultraviolet radiation in Nagqu is less than that in Lhasa. The results of UV-B radiation from several cities can be seen: the amount of ultraviolet radiation in the West and south of Tibet is higher than that in other regions.

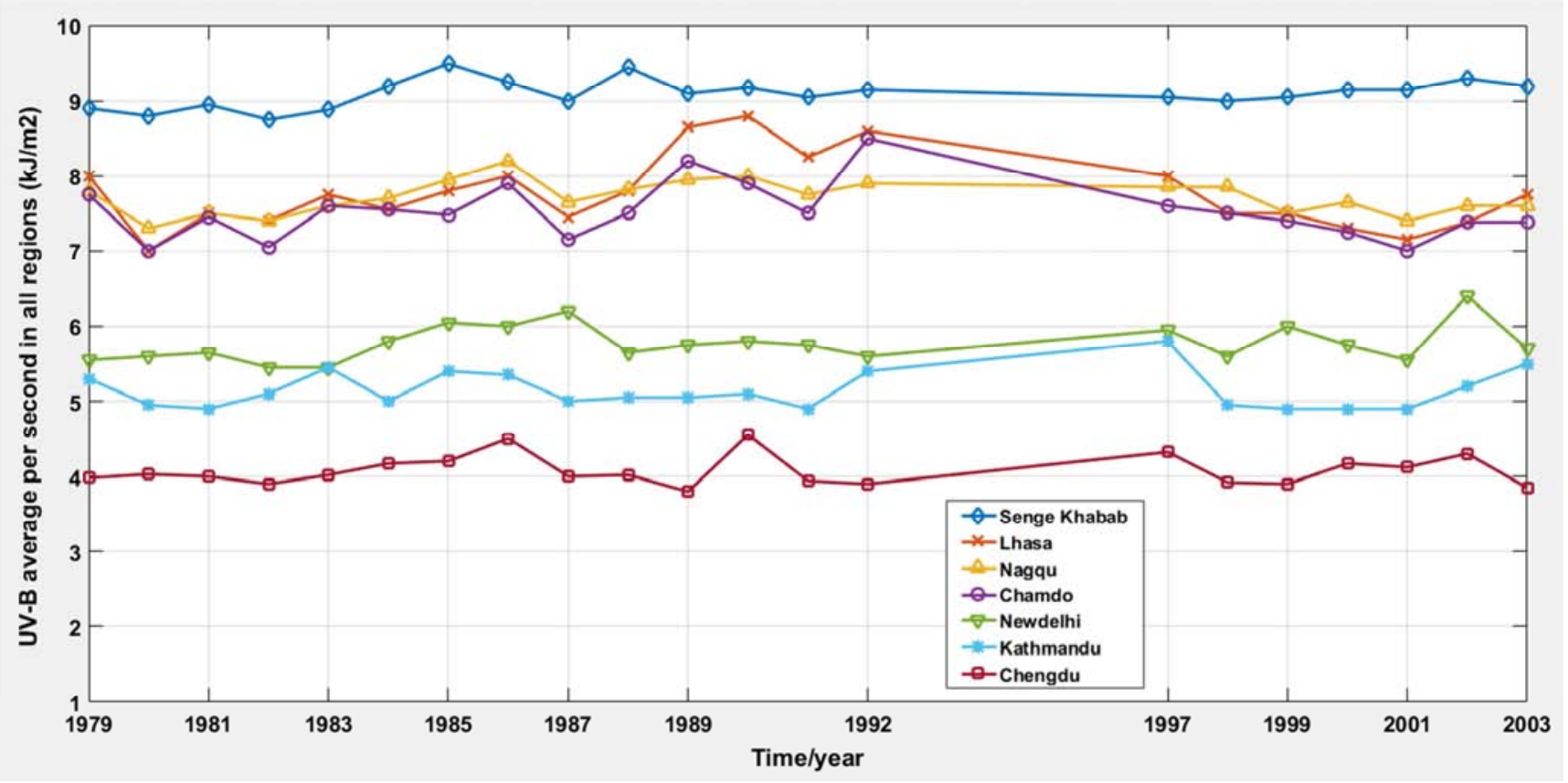

Fig. 2. Comparison of UV-B radiation in the surrounding cities of Lhasa and the Tibetan Plateau (May to August every year).

\subsection{Relationship Between Solar Ultraviolet Radiation and Altitude and Region in Tibet}

Based on the monitoring data, UV-B radiation stereo diagram 3 and UV-B radiation ichnography 4 of the Tibetan Plateau are presented. It can be seen from Figure 3 and Figure 4: There is a very close relationship between the UV-B radiation on the Qinghai Tibet Plateau and the elevation of the Tibetan Plateau.
The altitude is directly proportional to the ultraviolet radiation [5], [6] and the high radiation area of UV-B along the Himalaya Range and extend to the west of the eastern margin of the Gangdise Pamirs. This area is an important agricultural area in Tibet, and the density of highway network is high. The intensity of ultraviolet radiation has obvious relationship with the region. Tibet Chang Du area elevation is not high, but the ultraviolet 
intensity is much higher than the Tanggula Mountain Area [7]. The lowest UV radiation area is Linzhi's Tibet region [8], which is about the same as that of Qinghai.

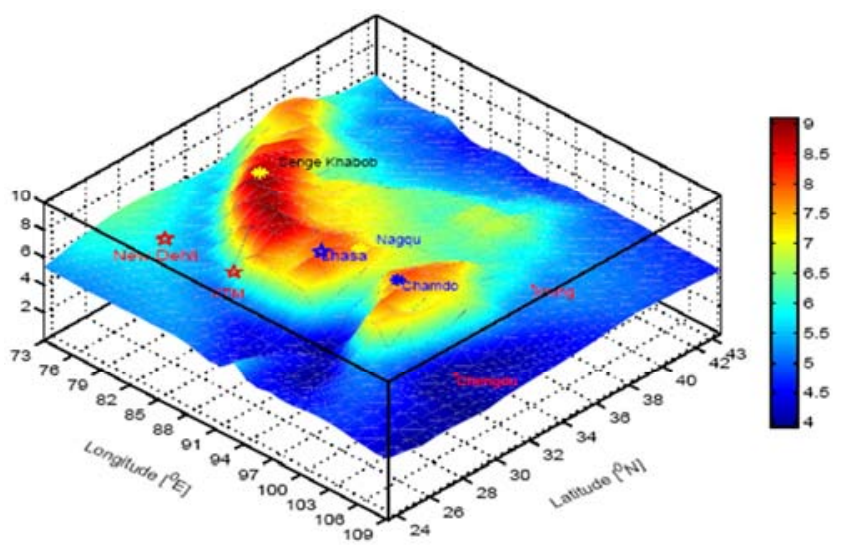

Fig. 3. Three dimensional map of $U V-B$ radiation on the Qinghai Tibet Plateau $\left(\mathrm{KJ} / \mathrm{m}^{2}\right)$.

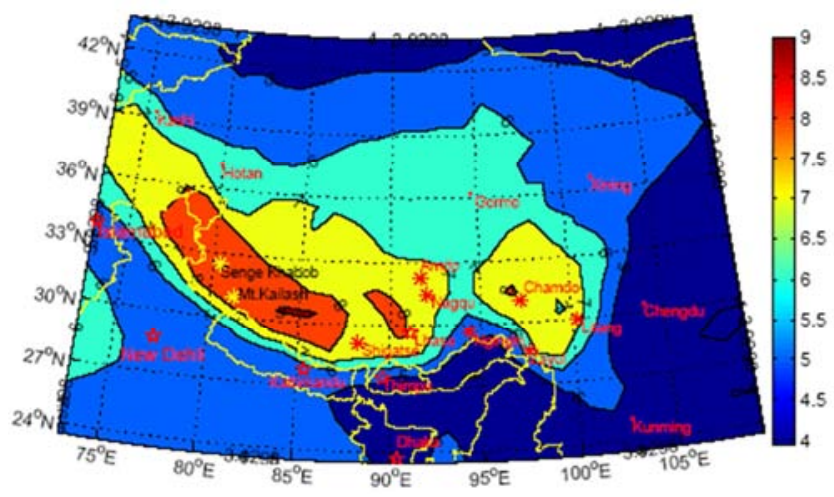

Fig. 4. UV-B radiation plan of the Tibetan Plateau $\left(\mathrm{KJ} / \mathrm{m}^{2}\right)$.

\section{Investigation and Disease Characteristics of Asphalt Pavement in Tibet}

Tibet asphalt pavement investigation involving Tibet and North Tibet and Tibetan southeast area. Road survey mainly distributed in Figure 4 shows the high radiation area (Figure 4 in yellow and red). The pavement diseases show distinct characteristics in the interior area. The disease is mainly as follows: pavement spalling, cracking, pavement and pavement white loose features, typical conditions as shown in figure 5.

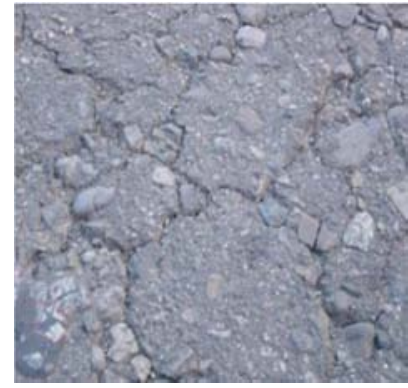

(1) Pavement spalling

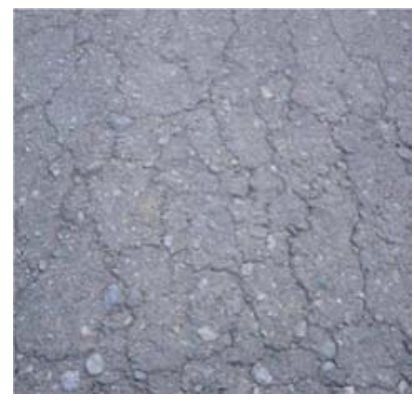

(2) Pavement cracking

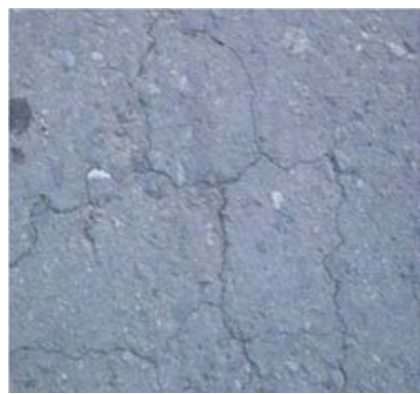

(3) The White Road

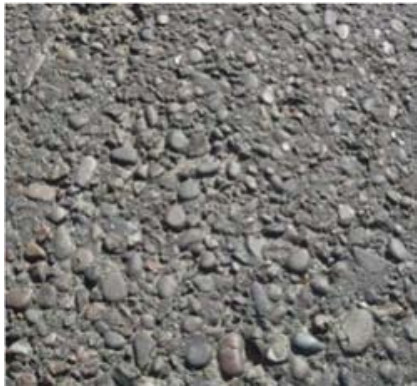

(4) The White Road

Fig. 5. Main failure modes of the west road surface.

\section{Analysis of the Relationship Between the Characteristics of Ultraviolet Radiation and the Damage of Asphalt Pavement}

In Tibet area, early damage of asphalt concrete pavement asphalt aging are closely related with ultraviolet rays, overload, frozen soil, freezing and thawing cycles and other climatic factors [9], [10]. The mechanism of ultraviolet radiation and pavement damage is discussed here.

The phenomenon of pavement become white is caused by asphalt aging. Because of the strong ultraviolet radiation in the northern part of Tibet, aging rate of Asphalt Pavement become faster. At the beginning, the newly paved asphalt pavement has the properties of black and shining. However, asphalt surface gradually lost luster after a period of use, from the original oil black turned to taupe, and then turned into offwhite. In the meanwhile, small cracks appear on the surface of asphalt. Under the combined action of vehicle load, temperature stress and rain water, these small cracks 
developed into a wide range of mesh crack gradually. These cracks (both small cracks and mesh crack) make the road become loose. Under the influence of rain, the phenomenon of water damage-pit is easy to take place.

The whole western part of Tibet, including Himalaya mountains and Kailash mountain, are all in the solar ultraviolet high radiation area. The radiation intensity is about three times as much as that of Jiangnan District, which are in the same latitude. The radiation intensity is also higher than the eastern part of Tibet. The strength of solar radiation in the western part of Tibet is strongest in the world. Because of the strong ultraviolet radiation, asphalt aging rate become faster. First, the color of asphalt fade, and become white gradually, then, the asphalt stiffen. This progress will take only one to two years in the course of the actual use of the road.

The general law of asphalt pavement damage in Tibet is as follow: ultraviolet radiation $\rightarrow$ the color of asphalt turn to white $\rightarrow$ Asphalt $\quad$ stiffening $\rightarrow$ small cracks appear $\rightarrow$ crack propagation $\rightarrow$ net $\quad$ cracking $\rightarrow$ seeper $\rightarrow$ asphalt $\quad$ spalling $\rightarrow$ pit appear. In the final analysis, the root cause of the law lies in the strong solar radiation. The strong ultraviolet radiation make asphalt aging faster, then the asphalt will lose its original performance, finally, under the influence of climate and traffic load, the pavement will be destroyed. So, we can come to the conclusion that strengthen anti-aging performance of the asphalt will greatly improve road's service performance.

\section{Conclusions}

Through the analysis of monitoring data of ultraviolet radiation in Tibet. We can get the distribution and intensity of the ultraviolet radiation in the Tibet plateau. The ultraviolet radiation in Tibet is strongest, not only in our country but also in the world. The strength of ultraviolet radiation in Lhasa area is two times than that of Beijing, and nearly 2.5 times than that of Chongqing. In terms of time, ultraviolet radiation has a certain periodicity, and change as the sine curve. Every year, June and July have the strongest ultraviolet radiation, then the ultraviolet radiation will weaken gradually. In December and January, the strength come to the weakest. After these two months, the strength begin to arise. In terms of space, the strength of ultraviolet radiation is related with altitude and location. Generally, the higher the altitude, the stronger the solar ultraviolet radiation. From the view of regional distribution, along Himalaya Range and Gangdise mountains and the Yarlung Zangbo River to the eastern margin of the Pamirs is the strong radiation zone. In addition, though the altitude of Chang $\mathrm{Du}$ area--which is in the eastern part of Tibet — is not very high, the ultraviolet radiation in these area is also very strong. On the whole, the area which has strong ultraviolet radiation is the area with high road density. The strong ultraviolet radiation accelerates the aging speed of asphalt pavement, then cause serious early damage of asphalt pavement.

Asphalt aging research results show that the phenomenon of aging and turn white take place after the completion of the second year. After that, these road disease will aggravate years after years. That one of the important characteristics which is different from other provinces, and strong ultraviolet radiation is the original important reason which make the asphalt pavement destroy.

\section{References}

[1] Liu Yang, Chen Zhengan. Analysis of solar radiation variations in Tibet during a short time [J]. Renewable Energy Resources, 2013, 06: $15-17+22$.

[2] Highway Bureau of Tibet Autonomous Region. Highway maintenance report of Tibet Highway Bureau 2006 [R]. Lhasa, 2006.

[3] Shen Jin-an. Pavement performance of asphalt and asphalt mixture [M]. Beijing, China Communications Press, 2001: 4-24, 185-200, 181-189.

[4] Wang Zhe. Discussion on evaluation method of anti aging property of high grade highway asphalt mixture [J]. HIGHWAY, 1997, (02): 34-44.

[5] G. Norsang, L. Kocbach, J. J. Stamnes, and Y. Gjessing. Spatial Distribution and Temporal Variation of UV and Ozone Over The Tibetan Plateau. 2004.

[6] Ma Xueqing. Estimation of solar radiation using NASA/POWER data [D]. Southwest University, 2015.

[7] Dai Jiaxi, Li Pengjie, and Su Hongde. To investigate the radiation condition in the Tanggula Mountain Area and the cold and heat source [C]. Qinghai Tibet Plateau meteorological Conference, Beijing: Meteorological research cooperation group of Tibetan Plateau, 1978: p 942.

[8] Wang Yi Li. The Study of Residential Indoor Thermal and Humid Environment in Tibet Area [M]. Chongqing University, 2015.

[9] Li Zheng. Study on Application of Polyphosphate and Rubber Composite Modified Asphalt and Its Mixture in the Tibet Region [J]. Highway Engineering, 2016, 01: 230-235+241.

[10] Ye Changyong, Xu Junda. Study on Anti Ultraviolet Aging Performance of SBS and SBR Modified Asphalt Mixture [J]. Journal of Wuhan University of Technology (Transportation Science \& Engineering), 2014, 04: 883-886. 